\title{
Tree-Transducers and Syntax-Connected Transductions
}

\author{
Peter Paul Schreiber \\ Technische Universität Berlin, Informatik PC2 \\ Ernst-Reuter-Platz 8, Berlin 10, West Germany
}

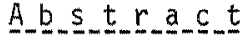

We investigate Finite Tree-Transducers operating top-down, bottom-up or both ways simultaneously. A comparative study of their transductional power is given. SyntaxConnected Transductions extending Syntax-Directed Transductions are investigated. Various types of transductions of local forests defined by Syntax-Connected Transduction Schemes can be performed by Finite Tree-Transducers.
\end{abstract}

\section{Introduction}

0perational automata like tree-transducers are extensions of classical automata. In addition to local processing like symbol-changing and state-switching, they can manipulate (permute, copy or erase) input-structures and output-structures. Finite state and push-down transducers have, so far, been very useful tools for designing and structuring the first phases of a compiler (such as the scanner and the parser). The more complicated phases consisting of semantic analysis, code generation and optimization, however, could not be supplied with such useful tools from automata theory. This is due to the fact that the objects to be dealt with in these phases are trees which have to be manipulated. As long as language translation had been understood as string processing and not as a tree-manipulating process, little effort was made to investigate machines which perform tree transductions. From the point of view of generalized automata theory, trees were used as inputs and (in a further generalization step) as outputs. Comparing these tree-transducers with syntax-directed transduction schemes performing transformations of the derivation trees of an underlying CF-Grammar, one can see that tree-transducers are more powerfut than the syntax-directed transduction schemes. Many tree-transforming phases of a compiler cannot be modelled by a syntax-directed transduction scheme, but by a tree-transducer. 


\section{Trees represented as terms}

To represent trees label led by elements of a set $\Sigma$ we use terms over $\Sigma$. The set $T_{\Sigma}$ of terms over $\Sigma$ is the smallest subset of $(\Sigma \mathcal{L}\{(,)\})^{*}$ satisfying:

$$
\begin{aligned}
& \text { (0) } \Sigma c T_{\Sigma} \\
& \text { (1) If } t_{1}, \ldots, t_{k} \varepsilon T_{\Sigma} \text { and } a \varepsilon \Sigma \text { then } a\left(t_{1} \ldots t_{k}\right) \varepsilon T_{\Sigma}
\end{aligned}
$$

Let $M$ be a set, $M_{n \Sigma}=\Phi$. The set $T_{\Sigma}[M]$ of terms over $\Sigma_{\text {indexed by } M}$ is the smallest subset of $\Sigma \cup M v\{()\},)^{*}$ such that

(0) $\Sigma_{U M C T}$

(1) If $t_{1}, \ldots, t_{k} \varepsilon T_{\Sigma}[M]$ for $k>0$ and $a \varepsilon \Sigma$ then $a\left(t_{1} \ldots t_{k}\right) \varepsilon T_{\Sigma}[M]$

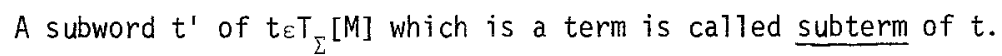

Notation: $t^{\prime} \leq t$. Two subterms $t^{\prime}$ and $t^{\prime \prime}$ of $t$ are independent iff $t^{\prime} \pm t^{\prime \prime}$ and $t^{\prime \prime} \leq t^{\prime}$.

Let $t^{\prime} \leq t$ and $r_{\varepsilon} T_{\Sigma}[M]$, then $t\left(t^{\prime}-r\right)$ is the term obtained by replacing $t^{\prime}$ by $r$.

Let $s_{1}, \ldots, s_{k}$ be pairwise independent subterms of $t$ and $\pi:[k] \rightarrow[k]$

$([k]=\{1, \ldots k\})$ any permutation and $r_{j} \varepsilon T_{\Sigma}[M] \quad(1 \leq i \leq k)$, then

$$
t\left(\left(s_{1}-r_{1}\right) \ldots\left(s_{k}-r_{k}\right)\right)=t\left(\left(s_{\pi(1)}-r_{\pi(1)}\right) \ldots\left(s_{\pi(k)}-r_{\pi(k)}\right)\right)
$$

Let $X=\left\{x_{i} \quad i \in \mathbb{N}\right\}$ be a set of parameters and $x_{k}=\left\{x_{1}, \ldots, x_{k}\right\}$.

The operation of simultaneous substitutions is defined as:

$$
t\left[t_{1}, \ldots, t_{k}\right]:=t\left(\left(x_{1}-t_{1}\right) \ldots\left(x_{k} \leftarrow t_{k}\right)\right)
$$

The frontier $\mathrm{fr}(\mathrm{t})$ of $t \varepsilon T_{\Sigma}[\mathrm{M}]$ is the word obtained by concatenating the labels of the leaves from left to right.

The depth $\|t\|$ of $t_{\varepsilon} T_{\Sigma}[M]$ is defined as:

$$
\|t\|=\left\{\begin{array}{l}
1 \text { for } t=a \varepsilon \Sigma \\
\max \left\|t_{i}\right\|+1 \text { for } t=a\left(t_{1} \ldots t_{k}\right) \varepsilon T_{\Sigma}[M]
\end{array}\right.
$$

\section{Finite Tree-Transducers}

A Finite Tree-Iransducer $(F T) P=(Q, \Sigma, \Delta, R, I)$ consists of a finite set $Q$ of states, an inputalphabet $\Sigma$, an outputalphabet $\Delta$, a finite set $R$ of rules and a subset I of $Q$ of distinguished states. 
A Top-Down-rule (T-rule) is a rule of the form:

$$
\langle q, a\rangle\left(x_{1} \ldots x_{k}\right) \rightarrow t \text { with }\left\langle q, a>\varepsilon Q \times \Sigma \text { and } t_{\varepsilon} T_{\Delta}\left[Q \times x_{k}\right]\right.
$$

or $\left\langle q, a>\longrightarrow t\right.$ with $t \in T_{\Delta}$.

T-rules of that type with $\|t\|=n$ are called $T(1, n)$-rules.

A T-ruie $\langle q, a\rangle\left(u_{1} \ldots u_{k}\right) \rightarrow t$ with $a\left(u_{1} \ldots u_{k}\right) \in T_{\Sigma}[X],\|t\|=n$

and $\quad\left\|a\left(u_{1} \ldots u_{k}\right)\right\|=m$ is called $T(m, n)$-rule.

A Top-Down-Finite-Tree-Transducer (TFT) is a FT with T-rules.

A move of a TFT is defined as a relation $\Gamma_{T}$ on $T_{\Sigma v \Delta v}(Q \times \Sigma)$.

Let $r, s \varepsilon T_{\Sigma \cup \Delta v(Q \times \Sigma)}$ and $R$ a set of $T(1,1)$-rules then $r F_{T} s$ iff

$\exists r^{\prime} \varepsilon T_{\Sigma \cup \cup}(Q \times \Sigma) \quad r^{\prime}=\langle q, a\rangle\left(t_{1} \ldots t_{k}\right) \leq r$

and $\exists\left(<q, a>\left(x_{1} \cdots x_{k}\right) \rightarrow t\right) \in R$

such that $s:=r\left(r^{\prime} \leftarrow t\left[t_{1}, \ldots, t_{k}\right]\right)$

- denotes the reflexive and transitive closure of - .

$T(P)=\left\{\langle r, s\rangle \varepsilon T_{\Sigma} \times T_{\Delta}\left|\left\langle q_{0}, r\right\rangle\right|-s_{\wedge} q_{0} \varepsilon I\right\}$ is called Tree-Transduction from $T_{\Sigma}$ to $T_{\Delta}$ performed by a TFT $P$.

A Bottom-Up-rule (B-rule) is a rule of the form

$$
a \rightarrow\langle t, p\rangle \text { with } a \varepsilon \Sigma, t_{\varepsilon} T_{\Delta} \text { and } p \varepsilon Q
$$

or $\quad a\left(\left\langle x_{1}, p_{1}>\ldots<x_{k}, p_{k}>\right) \rightarrow\left\langle t, p>\right.\right.$ with $a \varepsilon \Sigma, p, p_{1}, \ldots, p_{k} \varepsilon$ land $t \varepsilon T_{\Delta}\left[x_{k}\right]$.

$B$-rules of that type with $\|t\|=n$ are called $B(1, n)$-rules.

A B-ruie $a\left(u_{1} \ldots u_{k}\right) \rightarrow\langle t, p\rangle$ with $u_{i} \varepsilon T_{\Sigma}\left[X_{k} \times Q\right],\|t\|=n$

and $\quad\left\|a\left(u_{1} \ldots u_{k}\right)\right\|=m$ is called $B(m, n)$-rule.

A Bottom-Up-Finite-Tree-Transducer is a FT with B-rules.

A move of a BFT with $B(1,1)$-rules is defined as a relation $\Gamma_{B}$ on $T_{\Sigma u \Delta u(\Delta \times Q)}$.

Let $r, s \in T_{\sum v \Delta v(\Delta x Q)}$ then $r \vdash_{B} s$ iff

$$
\exists r^{\prime}=a\left(<t_{1}, p_{1}>\ldots<t_{k}, p_{k}>\right) \leq r
$$

and $\quad \exists\left(a\left(<x_{1}, p_{1}>\ldots<x_{k}, p_{k}>\right) \rightarrow\langle t, p>) \in R\right.$

such that $s:=r\left(r^{\prime} \ldots<t\left[t_{1}, \ldots, t_{k}\right], p>\right)$ 
$T(P)=\left\{\langle r, s\rangle \varepsilon T_{\Sigma} \times T T_{\Delta}|r| *\left\langle s, p_{0}>\wedge p_{0} E I\right\}\right.$ is the Tree-Transduction performed by a BFT $P$.

A rule is called rank-preserving if each parameter $x_{i}$ of its left side occurs on its right side.

A rule is called linear if each parameter $x_{i}$ of its left side occurs not more than once on its right side.

Rank-preserving rules can copy and do not erase subtrees while linear rules can erase and do not copy subtrees.

$$
\text { A FT with }\left\{\begin{array}{c}
\text { rank-preserving } \\
\text { linear }
\end{array}\right\} \text { rules is called }\left\{\begin{array}{l}
\text { RFT } \\
\text { LFT }
\end{array}\right\}
$$

and LRFT if its rules are linear and rank-preserving. A FT with $|\mathrm{Q}|=1$ is a pure FT

$$
{ }_{\Sigma} F T_{\Delta}=\left\{T(P)<T_{\Sigma} x T_{\Delta} I P \text { is a } F T\right\} \text { is called the class of } F \text {-Tree-Transductions }
$$

and we write FT for a fixed pair $(\Sigma, \Delta)$.

From now on we only consider Tree-Transducers with $T(1,1)$-rules or $B(1,1)$-rules.

Generalized Finite-Tree-Transducers are composed out of a

$$
\text { TFT } P_{T}=\left(Q_{T}, \Sigma, \Delta, R_{T}, I_{T}\right) \text { and a } B F T P_{B}=\left(Q_{B}, \Sigma, \Delta, R_{B}, I_{B}\right) \text {. }
$$

A move of a TBFT $P=\left(Q_{T}, Q_{B}, \Sigma, \triangle, R_{T}, R_{B}, I_{T}, I_{B}\right)$ is T-move followed by a $B$-move and a move of a BTFT $P=\left(Q_{B}, Q_{T}, \Sigma, \Delta, R_{B}, R_{T}, I_{B}, I_{T}\right)$ is a $B$-move followed by a $T$-move.

Let $P$ be a TBFT, then

$$
T(P)=\left\{\left\langle r, t>\varepsilon T_{\Sigma} \times T_{\Delta}\right|\left\langle q_{0}, r>F_{T} s \stackrel{*}{*}<t, p_{0}>, q_{0} \varepsilon I_{T} \wedge p_{0} \varepsilon I_{B}\right\}\right.
$$

and

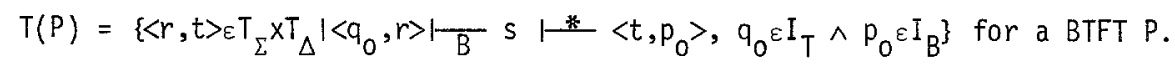

Theorem 1: For the classes of Finite Tree-Transductions the following lattice exists: (including results by ENGELFRIET, ROUNDS and THATCHER)

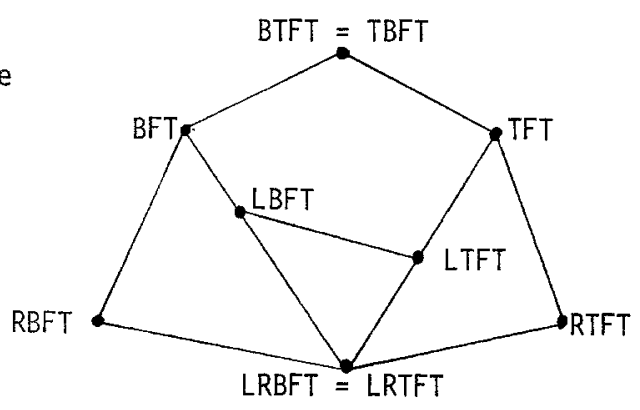




\section{Syntax-Connected-Transduction-Schemes}

Given a CF-Grammar $G=\left(\Sigma, \Sigma_{0}, P, S,\right)$ consisting of a finite alphabet $\Sigma$, subset $\Sigma_{0} c \Sigma$ of terminal symbols, a set $\operatorname{Pc}\left(\Sigma \backslash \Sigma_{0}\right) \times \Sigma^{+}$productions and a start symbol $S \varepsilon \Sigma \backslash \Sigma_{0}$. The elements of $\Sigma: \Sigma_{0}$ are called syntactic variables.

The set $D_{S}(G)$ of derivation trees of $G$ with root $S$ is defined as:

(0) $S_{E} D_{S}(G)$

(1) If $r \in D_{S}(G), f r(r)=w_{1} A w_{2}$ and $(A \rightarrow w) \varepsilon P$ with $w_{1}, w_{2} \varepsilon \Sigma^{*}, A \varepsilon \Sigma \backslash \Sigma_{0}$ then $r^{\prime}=r(A-A(W))$ is in $D_{S}(G)$

The set $D(G):=\left\{\operatorname{t\varepsilon D}_{S}(G) \mid f r(t) \varepsilon \Sigma_{0}^{+}\right\}$is called local forest of $G$.

A Syntax-Connected-Iransduction-Scheme (SCTS) $G=\left(G_{E}, G_{A}, k\right)$ consists of a CF-input grammar $G_{E}=\left(\Sigma, \Sigma_{0}, P_{E}, S\right)$, a CF-output grammar $G_{A}=\left(\Delta, \Delta_{0}, P_{A}, S\right)$ with $\Delta, \Delta_{0}<\Sigma<\Sigma_{0}$ and transduction rules $A \rightarrow w, v\left[i_{1}, \ldots, i_{m}\right]$, where $A \rightarrow w$ is an input production from $P_{E}, A \rightarrow v$ is an output production from $P_{A}$ such that $\left[i_{1}, \ldots, i_{m}\right] \varepsilon \kappa<\mathbb{N}^{m}$ connects positions of the common syntactic variables $i . e . A \rightarrow w, v\left[i_{1}, \ldots, i_{m}\right]$ has generally the following form:

$$
A \rightarrow g_{0} A_{1} g_{1} \ldots g_{k-1} A_{k} g_{k}, h_{0} A_{i_{1}} h_{1} \ldots h_{m-1} A_{j_{m}} h_{m}\left[i_{1}, \ldots, i_{m}\right]
$$

where $i_{j} \varepsilon[k]$ for $1 \leq j \leq m, \quad A_{i} \varepsilon \Sigma \backslash \Sigma_{0}, g_{j} \varepsilon \Sigma_{0}{ }^{*}(0 \leq i \leq k)$ and $h_{i} \varepsilon \Delta_{0}{ }^{*}(1 \leq i \leq m)$.

The set $T_{S}(G)$ of pairs of transduction trees is defined as:

(0) $\left\langle S, S>\in T_{S}(G)\right.$

(1) If $\langle r, s\rangle \varepsilon T_{\Sigma} \times T_{\Delta}$ such that $f r(r)=w_{0} B_{1} w_{1} \ldots w_{m-1} B_{m} w_{m}$ $f r(s)=v_{0} B_{i_{1}} v_{1} \ldots v_{n-1} B_{i_{n}} v_{n}$ and $\left[i_{1}, \ldots, i_{n}\right]$ with $i_{j} \varepsilon[m]$, $w_{i} \varepsilon \Sigma_{0}^{*}(0 \leq i \leq m), v_{i} \varepsilon \Delta_{0}^{*}(0 \leq i \leq n)$ then $\langle r, s\rangle \varepsilon T_{S}(G)$.

A relation $\rightarrow$ is defined on $T_{S}(G)$ as:

$$
\langle r, s\rangle \rightarrow\left\langle r^{\prime}, s^{\prime}\right\rangle
$$

iff there exists a transduction rule $B_{k} \rightarrow w, v\left[i_{1}, \ldots, i_{1}\right]$ such that:

1. $r^{\prime}=r\left(B_{k} \leftarrow B_{k}(w)\right)$

2. $s^{\prime}$ derives from $s$ by replacing all $B_{i_{j}}$ with $i_{j}=k$ by $B_{k}(v)$. or if no $i_{j}$ with $i_{j}=k$ exists $r^{\prime}=r\left(B_{k} \leftarrow B_{k}(w)\right)$ for $\left(B_{k} \rightarrow w\right) \varepsilon P_{E}$ and $s^{\prime}=s$. 
$T(G):=\left\{\langle r, s\rangle \varepsilon T_{S}(G) \mid f r(r) \varepsilon \Sigma_{0}^{+} f r(s) \varepsilon \Delta_{0}^{+}\right\}$is called Syntax-Connected-Transduction defined by $G$.

A transduction rule is called rank-preserving if each i $\varepsilon[k]$ appears at least once in $\left[i_{1}, \ldots, i_{m}\right]$ and linear if a $11 i_{j}(1 \leq i \leq m)$ are pairwise unequal in $\left[i_{1}, \ldots, i_{m}\right]$. If $\left[i_{1}, \ldots, i_{m}\right]=[1, \ldots, m]$ the transduction rule is called simple and regular for $\left[i_{1}\right]=[1]$.

A SCTS is a $\left\{\begin{array}{r}\text { RSDTS } \\ \text { SSDTS } \\ \text { LRSCTS } \\ \text { LSCTS } \\ \text { RSCTS }\end{array}\right\}$ if all transduction rules are $\left\{\begin{array}{l}\text { regular } \\ \text { simple } \\ \text { linear and rank-preserving } \\ \text { linear } \\ \text { rank-preserving }\end{array}\right\}$ $\Sigma_{\Sigma}^{S C T}=\left\{T(G) \times T_{\Sigma} \times T_{\Delta} \mid G\right.$ is a SCTS $\}$ is called the class of SC-Transductions and we write SCT for a fixed pair $(\Sigma, \Delta)$.

Theorem 2: For the classes of Syntaxconnected Transductions the following lattice exists:

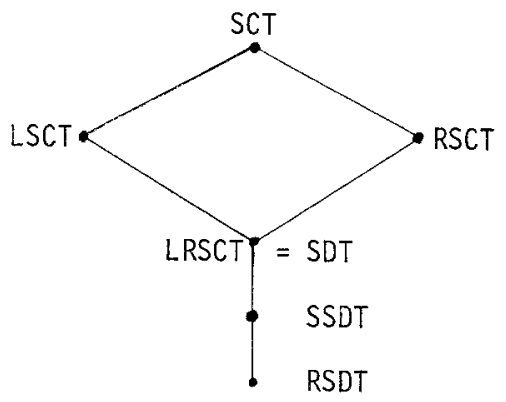

4. Relations between Schemes and Transducers

A SCT-Scheme defines a pair of local trees, while a tree-transducer operates on an input tree and produces an output tree.

Theorem 3: For each $\alpha$-SCTS $G$ exists a $\alpha$-TFT $P$ such that $T(G)=T(P)$. ( $\alpha=\mathrm{L}$ or $\mathrm{R}$ or $\mathrm{LR}$ )

This theorem implies several corollaries delivering a large variety of results dealing with special restricted cases for transducers and transduction schemes as well. 
Example: Let $G=\left(G_{E}, G_{A}, k\right)$ have the transduction rules:

$$
A \rightarrow a B A, A A d[2,2] \quad A \rightarrow a, c \quad(B \rightarrow b))_{E}
$$

The following pair of trees is in $T(G)$ :

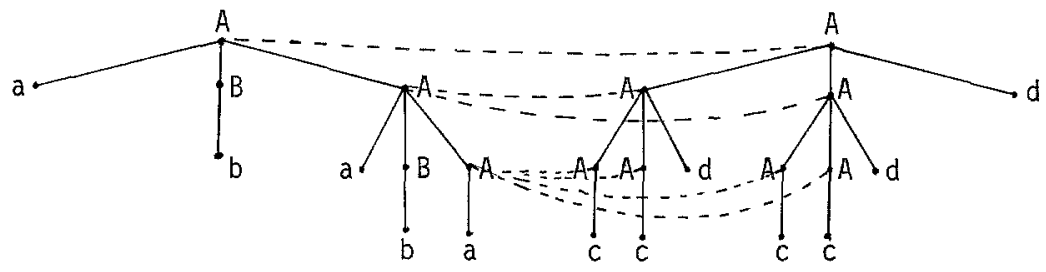

The dotted lines indicate the connections appearing in the course of generation.

This Tree-Transduction can be performed by a TFT with the following rules:

$$
\bar{A}\left(x_{1} x_{2} x_{3}\right) \rightarrow A\left(\bar{x}_{3} \bar{x}_{3} d\right) \quad \bar{A}(x) \rightarrow A(\bar{x}) \quad \bar{a} \rightarrow c
$$
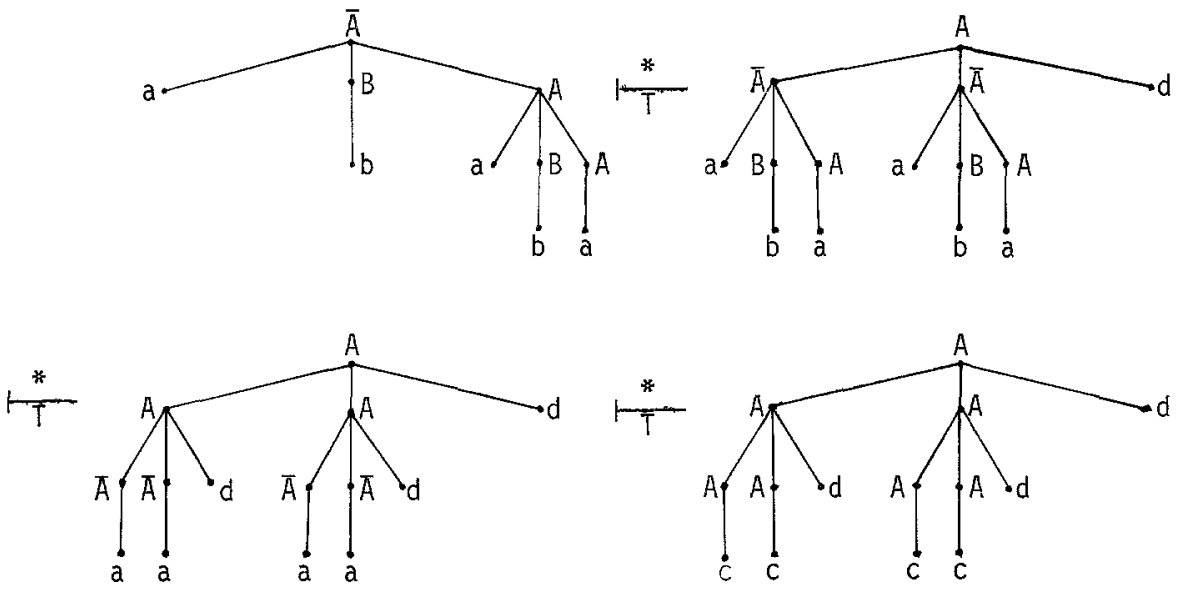

Acknowledgements:

I thank BLEICKE EGGERS for valuable discussions and GITA SARI for her excellent typing.

\section{References:}

ENGELFRIET, J.: Bottom-up and Top-Down Tree Transducers - a comparison.

Memorandum No. 19, 1971 Techn. Hogeschool Twente, Netherlands

ROUNDS, W.C.: Mappings and grammars on trees. MST 4, $257-287$ (1970)

SCHREIBER, P.P.: Baum-Transduktoren (Thesis forthcoming)

SCHREIBER, P.P.: Operational Automata for Compiler Design. Bericht Nr. $75-13$. Technische Universität Berlin, FB 20 - Kybernetik

THATCHER, J.W.: Generalized ${ }^{2}$ Sequential Machine Maps. JCSS 4, $339-367$ (1970) 